\title{
Accessing the single-particle structure of the Pygmy Dipole Resonance in ${ }^{208} \mathrm{~Pb}$
}

\author{
M. Spieker,,${ }^{1, *}$ A. Heusler, ${ }^{2}$ B. A. Brown,${ }^{3,4}$ T. Faestermann, ${ }^{5}$ R. Hertenberger,${ }^{6}$ G. \\ Potel, ${ }^{7}$ M. Scheck, ${ }^{8,9}$ N. Tsoneva, ${ }^{10}$ M. Weinert, ${ }^{11}$ H.-F. Wirth, ${ }^{6}$ and A. Zilges ${ }^{11}$ \\ ${ }^{1}$ Department of Physics, Florida State University, Tallahassee, Florida, 32306, USA \\ ${ }^{2}$ Niebuhr-Str. 19c, D-10629 Berlin, Germany \\ ${ }^{3}$ National Superconducting Cyclotron Laboratory, Michigan State University, East Lansing, Michigan 48824, USA \\ ${ }^{4}$ Department of Physics and Astronomy, Michigan State University, East Lansing, Michigan 48824, USA \\ ${ }^{5}$ Physik Department, Technische Universität München, D-85748 Garching, Germany \\ ${ }^{6}$ Fakultät für Physik, Ludwig-Maximilians-Universität München, D-85748 Garching, Germany \\ ${ }^{7}$ Lawrence Livermore National Laboratory, Livermore, California, 94550, USA \\ ${ }^{8}$ School of Computing, Engineering, and Physical Sciences, \\ University of the West of Scotland, Paisley, PA1 2BE,UK \\ ${ }^{9}$ SUPA, Scottish Universities Physics Alliance, UK \\ ${ }^{10}$ Extreme Light Infrastructure (ELI-NP), Horia Hulubei National Institute of Physics \\ and Nuclear Engineering (IFIN-HH), RO-077125, Bucharest-Măgurele, Romania \\ ${ }^{11}$ Institut für Kernphysik, Universität zu Köln, Zülpicher Straße 77, D-50937 Köln, Germany
}

(Dated: September 3, 2020)

\begin{abstract}
New experimental data on the neutron single-particle character of the Pygmy Dipole Resonance $(\mathrm{PDR})$ in ${ }^{208} \mathrm{~Pb}$ are presented. They were obtained from $(d, p)$ and resonant proton scattering experiments performed at the Q3D spectrograph of the Maier-Leibnitz Laboratory in Garching, Germany. The new data are compared to the large suite of complementary, experimental data available for ${ }^{208} \mathrm{~Pb}$ and establish $(d, p)$ as an additional, valuable, experimental probe to study the PDR and its collectivity. Besides the single-particle character of the states, different features of the strength distributions are discussed and compared to Large-Scale-Shell-Model (LSSM) and energy-density functional (EDF) plus Quasiparticle-Phonon Model (QPM) theoretical approaches to elucidate the microscopic structure of the PDR in ${ }^{208} \mathrm{~Pb}$.
\end{abstract}

Atomic nuclei with large proton-neutron asymmetry, like ${ }^{208} \mathrm{~Pb}$, form a neutron skin [1]. The neutron-skin thickness, $\Delta r_{n p}$, is directly correlated to properties of neutron stars [1 5]. This raised the interest of the science community in determining it experimentally [69]. Following the first multi-messenger detection of a binary neutron star merger [10] including gravitational waves [1], this interest has been recently reinforced [12].

The electric dipole polarizability $\alpha_{D}[6,8,8,13,19]$ is one key observable investigated to obtain constraints on $\Delta r_{n p}$. For its precise determination, the low-lying $E 1$ strength is extremely important. The term "Pygmy Dipole Resonance" (PDR) has been commonly used for the E1 strength around and below the neutronseparation energy, $S_{n}$ 20 24]. The PDR strength might also correlate more strongly with $\Delta r_{n p}[18,25,29]$ and, thus, provide tighter constraints. However, the possibly stronger correlation has been critically discussed [30 33]. In any case, it would be necessary to distinguish the PDR from other $E 1$ modes like the low-energy tail of the Giant Dipole Resonance (GDR) (see, e.g., [17, 34 37]). It has been shown that the PDR strength strongly impacts neutron-capture rates in the $s$ and $r$ process 17, 38 41]. A precise understanding of its microscopic structure is also essential to pin down how the PDR contributes to the $\gamma$-ray strength function ( $\gamma \mathrm{SF})$ often used to calculate $(n, \gamma)$ rates [41], i.e. whether there is a dependence of the $\gamma$ SF's shape on excitation energy, spin-parity quantum number or even specific nuclear structure 42 49].
Depending on the mass region of the nuclear chart, the low-lying $E 1$ response to isovector and isoscalar probes, or to probes testing surface rather than bulk properties, is different [34, 36, 50 60]. While in lighter nuclei usually state-to-state differences were observed, some heavier nuclei featured the so-called isospin splitting of the low-lying $E 1$ response (see the review articles [22, 24]). These different responses emphasized that different underlying structures would indeed need to be disentangled experimentally, if stringent comparisons to microscopic models wanted to be made.

Besides its isospin structure, the degree of collectivity of the PDR is still under debate 24, 61 70. Often, collectivity is accessed in terms of the number of oneparticle-one-hole $(1 \mathrm{p}-1 \mathrm{~h})$ excitations acting coherently and, therefore, causing enhanced transition strength 64 66]. A recent theoretical study of the PDR in ${ }^{68} \mathrm{Ni}[24]$, which used a fully self-consistent nonrelativistic meanfield approach based on Skyrme Hartree-Fock plus random phase approximation ( $\mathrm{HF}+\mathrm{RPA})$, reinforced that coherence between several $1 \mathrm{p}-1 \mathrm{~h}$ configurations is rather observed in the isoscalar than in the isovector channel. Qualitatively comparable results had been obtained for ${ }^{132} \mathrm{Sn}$ and ${ }^{208} \mathrm{~Pb}$ by employing similar theoretical approaches [65, 66]. These theoretical results question the usefulness of studying the PDR's collectivity based on the isovector $E 1$ strengths alone.

In this work, we present a detailed, high-resolution $(d, p)$ experimental study of the PDR in ${ }^{208} \mathrm{~Pb}$ and com- 
plement it with available experimental data to discuss the PDR's microscopic structure and its influence on experimental observables by comparing to state-of-theart, theoretical models. The neutron $1 \mathrm{p}-1 \mathrm{~h}$ configurations contributing to forming the PDR are accessed from $(d, p)$ data up to the proton-separation energy, $S_{p}$, and, for a limited number of states, from the results of resonant proton scattering via isobaric analog resonances $\left(\left(p, p^{\prime}\right)_{\mathrm{IAR}}\right)$ [1] 76], which probes components that could not be populated in the selective one-neutron transfer reaction. An unprecedented access to the theoretical wave functions was achieved.

When discussing its collectivity within the HF + RPA approach, Roca-Maza et al. identified the PDR of ${ }^{208} \mathrm{~Pb}$ above $7 \mathrm{MeV}$ [65]. Following a comparison of Nuclear Resonance Fluorescence data and Quasiparticle-Phonon Model (QPM) calculations, Ryezayeva et al. had argued that the PDR should indeed correspond to the strength observed around $S_{n}$ [62]. The lower-lying $1^{-}$ states should have a more pure single-particle character [62]. Poltoratska et al. 35] considered, however, all low-lying $E 1$ strength up to $\sim 8.3 \mathrm{MeV}$ to belong to the PDR in agreement with a $\left({ }^{17} \mathrm{O},{ }^{17} \mathrm{O}^{\prime} \gamma\right)$ experiment [54], performed to study its isospin character. Based on QPM calculations for ${ }^{206} \mathrm{~Pb}$, dominantly the neutron 1p-1h states below $S_{n}$ were identified to belong to the PDR of the $N=124 \mathrm{~Pb}$ isotope [17]. The importance of including two-particle-two-hole (2p-2h) configurations to describe the isovector $B(E 1)$ strength fragmentation was pointed out in 77] using Large-Scale-Shell-Model (LSSM) calculations [78]. Also the possibility of tetrahedral configurations in ${ }^{208} \mathrm{~Pb}$ was presented and some of the lower-lying states, including the $1_{1}^{-}$state, were discussed to originate from this exotic type of excitation 79$]$.

The new data, presented here, were obtained from a series of experiments performed to study excited states in ${ }^{208} \mathrm{~Pb}$ with the high-resolution Q3D spectrograph of the Maier-Leibnitz Laboratory (MLL) in Garching, Germany 80,81$]$. For the $(d, p)$ experiments, the deuterons were accelerated to $22 \mathrm{MeV}$ and impinged onto a 0.11 $\mathrm{mg} / \mathrm{cm}^{2}$ thick, highly-enriched ${ }^{207} \mathrm{~Pb}$ target (99\% enrichment) on a Carbon backing. After the reaction, the residual particles were momentum-analyzed with the Q3D and detected in the focal-plane detection system [82, 83]. By adjusting the horizontal entrance slits, half $\left( \pm 1.6^{\circ}\right)$ of the Q3D's maximum angular acceptance was used and an energy resolution of better than $6 \mathrm{keV}$ (FWHM) achieved. This facilitated the analysis of the dense excitation spectra seen in Fig. 1.

The $(d, p)$ data were analyzed at three scattering angles; $20^{\circ}, 25^{\circ}$, and $30^{\circ}$. This allows to distinguish the two different transfer configurations through which the known $1^{-}$states of ${ }^{208} \mathrm{~Pb}$ [85, 86] can be populated from the $J^{\pi}=1 / 2^{-}$ground state of ${ }^{207} \mathrm{~Pb}$; namely $\left(3 \mathrm{p}_{1 / 2}\right)^{-1}\left(4 \mathrm{~s}_{1 / 2}\right)^{+1}(l=0)$ and $\left(3 \mathrm{p}_{1 / 2}\right)^{-1}\left(3 \mathrm{~d}_{3 / 2}\right)^{+1}(l=$ 2). The angular distributions are shown in Fig. 2 along-

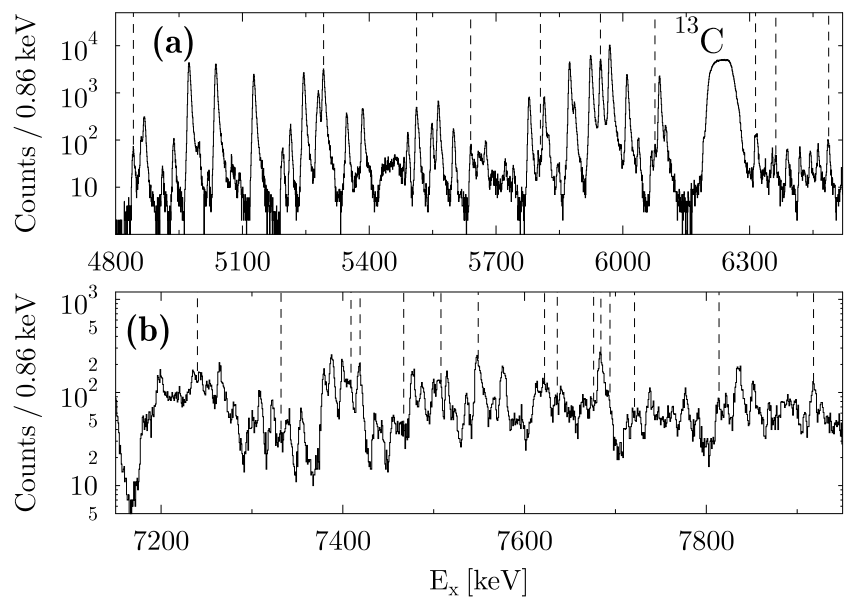

Figure 1. (a), (b) ${ }^{207} \mathrm{~Pb}(d, p){ }^{208} \mathrm{~Pb}$ spectra taken at $\theta=25^{\circ}$ for two different magnetic settings. Only a part of the spectrum is shown in panel (b). Contamination from the ${ }^{12} \mathrm{C}(d, p){ }^{13} \mathrm{C}$ reaction is observed (labeled with ${ }^{13} \mathrm{C}$ ) due to the Carbon backing of the target. The kinematic correction with the Q3D multipole element was applied to the ${ }^{207} \mathrm{~Pb}(d, p)$ reaction causing the peaks observed from ${ }^{12} \mathrm{C}(d, p)$ to be significantly broader (compare 84]). Known $J^{\pi}=1^{-}$states of ${ }^{208} \mathrm{~Pb}$ [85 87, which could be resolved, are highlighted with vertical, dashed lines. All other states, seen in the spectra, correspond to excited states of ${ }^{208} \mathrm{~Pb}$ with a $3 \mathrm{p}_{1 / 2}$ neutronhole component in their wave function. Below $S_{n}$, many of them were experimentally observed before [85, 86, 88 90 ].

side Distorted-Wave-Born-Approximation (DWBA) calculations performed with the coupled-channels program CHUCK3 91. The global optical-model parameters (OMP) of [92] were used for the protons and of [93] for the deuterons with adjustments to the real potential of the volume Woods-Saxon part from [94]. With the exception of using an effective neutron-separation energy for states above $S_{n}$, the same OMP were used for all excited states. As shown in Fig.2 the measured and DWBA angular distributions are in excellent agreement. The dominant contributions of the most strongly excited $1^{-}$states at $5292 \mathrm{keV}, 5512 \mathrm{keV}$, and $5947 \mathrm{keV}$ were previously identified 71, 72, 88 90, 95] and confirmed here. Only small additional $\left(3 \mathrm{p}_{1 / 2}\right)^{-1}\left(4 \mathrm{~s}_{1 / 2}\right)^{+1}$ contributions were needed to explain the experimental angular distributions for the $5512-\mathrm{keV}$ and $5947-\mathrm{keV}$ states. In return, this highlights the sensitivity of the present experiment to such small contributions. The $\left(p, p^{\prime}\right)_{\mathrm{IAR}}$ data on the $3 d_{3 / 2}$ and $4 s_{1 / 2}$ IARs in ${ }^{209} \mathrm{Bi}$ confirm the dominant structure assignments for the $5292-\mathrm{keV}$ and $5947-\mathrm{keV}$ state [compare Fig.3(b)], respectively. Also at higher excitation energies, superpositions of the two configurations were often needed to explain the experimental $(d, p)$ data as shown for three examples in Fig.2, As indicated by the $\left(p, p^{\prime}\right)_{\text {IAR }}$ data, other $1 \mathrm{p}-1 \mathrm{~h}$ configurations are important as well and might dominate the structure of the states [compare Fig.3(b)]. In total, 11 out of the 15 ampli- 


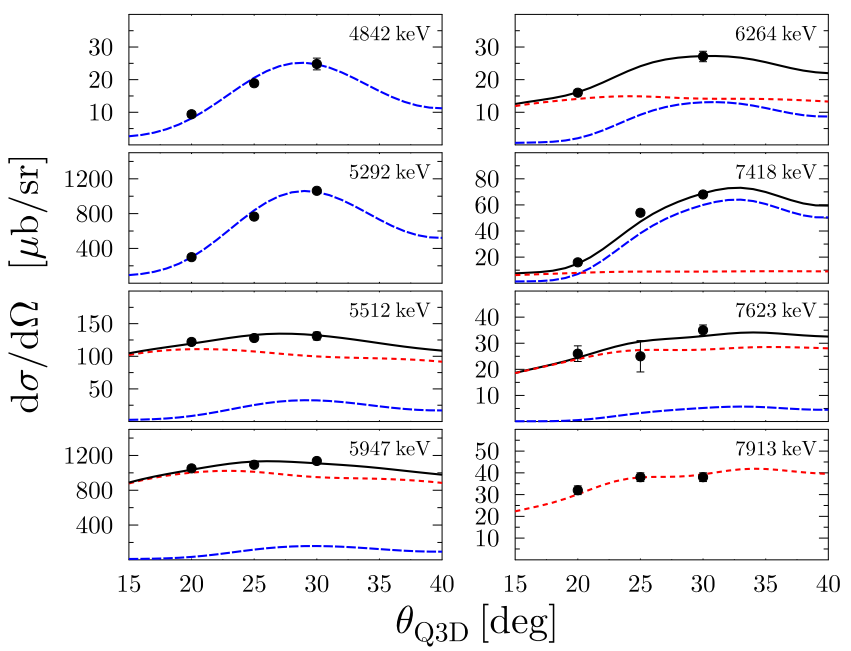

Figure 2. (color online) Measured $(d, p)$ angular distributions (differential cross sections $d \sigma / d \Omega$ ) for selected $J^{\pi}=1^{-}$ states (circles) in comparison to DWBA calculations (lines). Two different $1 \mathrm{p}-1 \mathrm{~h}$ configurations, $\left(3 \mathrm{p}_{1 / 2}\right)^{-1}\left(4 \mathrm{~s}_{1 / 2}\right)^{+1}$ (blue, longer dashed lines) and $\left(3 \mathrm{p}_{1 / 2}\right)^{-1}\left(3 \mathrm{~d}_{3 / 2}\right)^{+1}$ (red, shorter dashed lines), have been assumed to describe the experimental distributions. Black, solid lines correspond to superpositions of these two individual configurations. No multistep transfer was considered. For states above $S_{n}$, an effective neutronseparation energy of $S_{n}=8.5 \mathrm{MeV}$ had to be used. Otherwise, the shape of the angular distribution would have been heavily distorted. A similar approach had been chosen in [96]. The unique features of the $l=0$ and $l=2$ transfers remain unchanged. For the $1^{-}$state at $6264 \mathrm{keV}$, a Carbon contaminant prevented a cross-section measurement at $\theta=25^{\circ}$.

tudes were studied experimentally 71 76]. More details on the determination of the relative $c_{L J l j}$ amplitudes for the different neutron $1 \mathrm{p}-1 \mathrm{~h}$ configurations from $\left(p, p^{\prime}\right)_{\mathrm{IAR}}$ are presented in 71 75, 87].

The model-indepedent, angle-integrated $(d, p)$ cross sections and $c_{L J l j}$ amplitudes from $\left(p, p^{\prime}\right)_{\text {IAR }}$ are shown in Fig. [3in comparison to a selection of other experimental data on the PDR in ${ }^{208} \mathrm{~Pb}$ 35, 54]. The $(d, p)$ strength pattern [Fig. [3.(a)] is dominated by the two strongly populated $1^{-}$states at $5292 \mathrm{keV}$ and $5947 \mathrm{keV}$, corresponding to the major fragments of the $\left(3 \mathrm{p}_{1 / 2}\right)^{-1}\left(4 \mathrm{~s}_{1 / 2}\right)^{+1}[S=$ $0.77(4)]$ and $\left(3 \mathrm{p}_{1 / 2}\right)^{-1}\left(3 \mathrm{~d}_{3 / 2}\right)^{+1}[S=0.66(4)]$ neutron 1p-1h strength (compare Fig.22), respectively. The stated spectroscopic factors, $S$, are model-dependent but were determined consistently, i.e. using the same OMP. This is different from the approach chosen in 90, 95], where OMP were varied depending on the $l$ transfer introducing a stronger model dependency. While the $5292-\mathrm{keV}$ state shows appreciable $B(E 1)$ strength [35, 62] and is also comparably strongly populated in $\left({ }^{17} \mathrm{O},{ }^{17} \mathrm{O}^{\prime} \gamma\right)$ 54], the $5947-\mathrm{keV}$ state is, strikingly, barely excited with the electromagnetic probe and not at all with the hadronic probe [compare Figs.3](a), (c), (d)]. Remarkably, the group of states with excitation energies of $6264 \mathrm{keV}$,
$6314 \mathrm{keV}, 6362 \mathrm{keV}$, and $6486 \mathrm{keV}$ features both gradually decreasing $(d, p)$ cross sections and isovector $B(E 1)$ strengths, while only the $6264-\mathrm{keV}$ state is strongly excited in $\left({ }^{17} \mathrm{O},{ }^{17} \mathrm{O}^{\prime} \gamma\right)$. For the $6264-\mathrm{keV}$ and $6314-\mathrm{keV}$ states, mixtures of $l=0$ and $l=2$ transfers were needed to describe the experimental angular distributions (see, exemplary, the 6264-keV state in Fig.(2). One configuration was sufficient for the $6362-\mathrm{keV}(l=0)$ and 6486 $\mathrm{keV}(l=2)$ states. Interestingly, the $6264-\mathrm{keV}$ state is the only one of the four, which has $\left(2 f_{5 / 2}\right)^{-1}\left(2 g_{7 / 2}\right)^{+1}$ and $\left(2 \mathrm{f}_{7 / 2}\right)^{-1}\left(2 \mathrm{~g}_{7 / 2}\right)^{+1}$ components in its wave function [compare Fig.[3](b)].

Figs. 3(e)-(m) presents the results of LSSM 78] and energy-density functional (EDF)+QPM [97] calculations. To calculate the differential cross sections $d \sigma / d \Omega$, predicted spectroscopic factors, i.e. the overlap of the ${ }^{207} \mathrm{~Pb}$ ground state with excited $1^{-}$states in ${ }^{208} \mathrm{~Pb}$ when adding a neutron, were combined with the DWBA calculations [91], which described the experimental data. The angle-integrated $\sigma_{(d, p)}$ cross sections were also determined between $\theta=20^{\circ}-30^{\circ}$.

The LSSM calculations [Fig. 3)(e)-(i)] were introduced in [77, 78]. In addition to the $\sigma_{(d, p)}$ values (see supplement for truncation at $1 \mathrm{p}-1 \mathrm{~h}$ level [98]), we provide the decomposition of the wave functions into the different neutron $1 \mathrm{p}-1 \mathrm{~h}$ components $(>1 \%)$ relative to the total wave function $\psi_{\text {total }}$ [Fig.3(f)], and the contributions of $1 \mathrm{p}-1 \mathrm{~h}$ and $2 \mathrm{p}-2 \mathrm{~h}$ components to $\psi_{\text {total }}$ [compare Fig.3(g)]. The predicted excitation energy, $5226 \mathrm{keV}$, of the major $\left(3 \mathrm{p}_{1 / 2}\right)^{-1}\left(4 \mathrm{~s}_{1 / 2}\right)^{+1}\left[S_{L S S M}=0.56\right]$ fragment is very close to the experimental $5292-\mathrm{keV}$ state $\left[S_{\text {exp }}=0.77(4)\right]$. Considering the other strong fragment with $S_{L S S M}=0.16$ at $5469 \mathrm{keV}$ provides a centroid energy of $5280 \mathrm{keV}$ with $S_{L S S M}=0.72$ in almost perfect agreement with the experimental data. Below 6.25 MeV, the $\left(3 \mathrm{p}_{1 / 2}\right)^{-1}\left(3 \mathrm{~d}_{3 / 2}\right)^{+1}$ strength is much more fragmented than experimentally observed. The strongest fragment is predicted at $6171 \mathrm{keV}$ with $S_{L S S M}=0.34$. The LSSM centroid of the $l=2$ strength is found at $5912 \mathrm{keV}$ with $S_{L S S M}=0.78\left(E_{x}<6.25 \mathrm{MeV}\right)$, which again compares well to the experimental centroid at $5904 \mathrm{keV}\left[S_{\text {exp }}=0.73(4)\right]$ when considering the 5512 $\mathrm{keV}$ and $5947-\mathrm{keV}$ states. The summed, angle-integrated cross sections below $S_{n}$ are $\sum \sigma_{(d, p)_{e x p}}=1524(17) \mu \mathrm{b}$ and $\sum \sigma_{(d, p)_{L S S M}}=1470 \mu \mathrm{b}$. However, the fragmentation of the LSSM spectroscopic strength between $S_{n}$ and $S_{p}$ is not as observed in experiment. For firm $1^{-}$states above $S_{n}, \sum \sigma_{(d, p)_{\text {exp }}}$ is $254(9) \mu \mathrm{b}$ while the LSSM predicts only $22 \mu \mathrm{b}$. $13 \%$ of the $d_{3 / 2}$ and $9 \%$ of the $s_{1 / 2}$ strength are pushed to energies higher than $8.6 \mathrm{MeV}$ in the LSSM. The data suggest that this strength is located below $S_{p}$.

Many neutron $1 \mathrm{p}-1 \mathrm{~h}$ excitations contribute to $\psi_{\text {total }}$ with the strongest component never exceeding $56 \%$. Given the experimental limitation of only being able to determine three to four amplitudes when studying one 


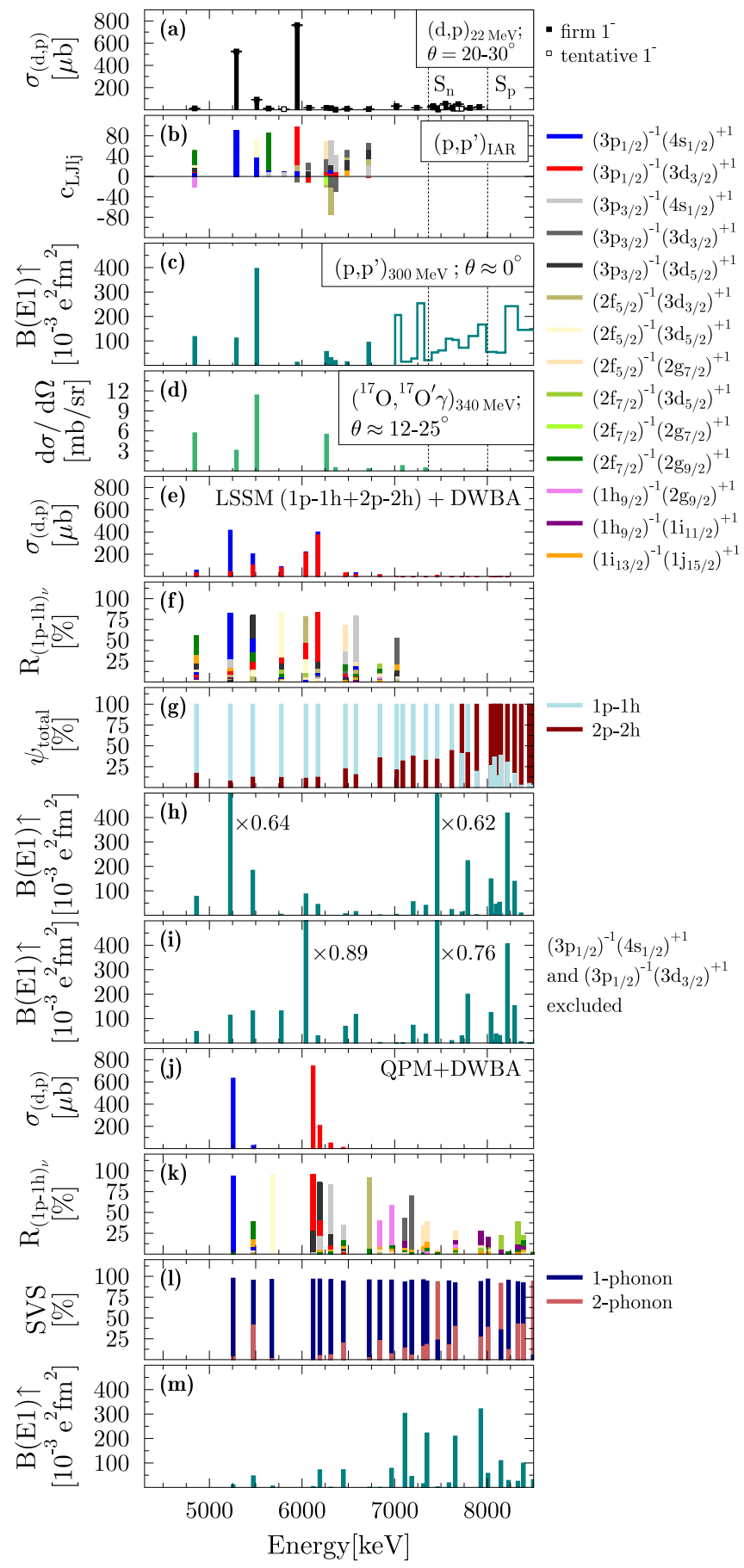

Figure 3. (color online) (a) Angle-integrated $(d, p)$ cross sections $\sigma_{(d, p)}$, (b) $c_{L J l j}$ amplitudes from $\left(p, p^{\prime}\right)_{\text {IAR }} 71,72,75$, 76], (c) isovector $B(E 1)$ strengths from $\left(p, p^{\prime}\right)$ 35], and (d) differential cross sections from $\left({ }^{17} \mathrm{O},{ }^{17} \mathrm{O}^{\prime} \gamma\right)[54]$. The latter probe the isoscalar character of the $1^{-}$states [54]. (e) $\sigma_{(d, p)}$ predicted by combining LSSM spectroscopic factors with DWBA calcultions. (f) Decomposition of the LSSM wave functions into neutron $1 \mathrm{p}-1 \mathrm{~h}$ components relative to the total wave function $\psi_{\text {total }}$. (g) $1 \mathrm{p}-1 \mathrm{~h}$ and $2 \mathrm{p}-2 \mathrm{~h}$ contributions to $\psi_{\text {total }}$. LSSM isovector $B(E 1)$ strength predicted when (h) including all or (i) exluding the specified contributions. (j)(m) same as (e)-(h) but for EDF+QPM. SVS stands for "state-vector structure" 97, 98].
IAR, this seems largely consistent with the $\left(p, p^{\prime}\right)_{\text {IAR }}$ data. As seen from the comparison of Figs. $3(\mathbf{b})$ and 3(f), most $1^{-}$states cannot be considered as simple neutron $1 \mathrm{p}-1 \mathrm{~h}$ states. The $(d, p)$ data prove that almost all $1^{-}$states have at least small $\left(3 \mathrm{p}_{1 / 2}\right)^{-1}\left(4 \mathrm{~s}_{1 / 2}\right)^{+1}$ and $\left(3 \mathrm{p}_{1 / 2}\right)^{-1}\left(3 \mathrm{~d}_{3 / 2}\right)^{+1}$ components, many of which were below the sensitivity limit in $\left(p, p^{\prime}\right)_{\text {IAR }}$. With only a few exceptions, the neutron $1 \mathrm{p}-1 \mathrm{~h}$ contribution makes up around $80 \%$ of $\psi_{\text {total }}$ in the LSSM at lower energies (compare $R_{(1 p-1 h)_{\nu}}$ in Fig.3(f) for the first ten $1^{-}$ states). The strongest neutron $1 \mathrm{p}-1 \mathrm{~h}$ component in the wave function of the $1_{1}^{-}$is identified as $\left(2 \mathrm{f}_{7 / 2}\right)^{-1}\left(2 \mathrm{~g}_{9 / 2}\right)^{+1}$ in both $\left(p, p^{\prime}\right)_{\text {IAR }}$ and the LSSM. The experimental data for the $1_{1}^{-}$support that less than $60 \%$ of $\psi_{\text {total }}$ are due to neutron $1 \mathrm{p}-1 \mathrm{~h}$ components 74,79 . For almost all lower-lying $1^{-}$states, the $2 \mathrm{p}-2 \mathrm{~h}$ contribution already exceeds $10 \%$ [compare Fig. 3(g)]. A clear structural change is observed above $7.5 \mathrm{MeV}$, where $2 \mathrm{p}-2 \mathrm{~h}$ configurations begin to dominate the wave functions. Note that Poltoratska et al. [35] experimentally observed a structure change at $\sim 8.2 \mathrm{MeV}$, where GDR-type wave functions began to dominate. At $\sim 8.4 \mathrm{MeV}$, the $1 \mathrm{p}-1 \mathrm{~h}$ contribution to $\psi_{\text {total }}$ drops well below $10 \%$ in the LSSM.

The LSSM $B(E 1)$ strength distribution [77] is shown in Fig.3(h). Problematically, the most enhanced $B(E 1)$ value is observed for the major $\left(3 \mathrm{p}_{1 / 2}\right)^{-1}\left(4 \mathrm{~s}_{1 / 2}\right)^{+1}$ fragment, i.e. the $1_{2}^{-}$state in conflict with experiment [compare Fig.[3(c)]. The major $\left(3 \mathrm{p}_{1 / 2}\right)^{-1}\left(3 \mathrm{~d}_{3 / 2}\right)^{+1}$ fragment has an experimental $B(E 1)=13(1) \times 10^{-3} \mathrm{e}^{2} \mathrm{fm}^{2}$. The LSSM calculations predict $B(E 1)=45 \times 10^{-3} \mathrm{e}^{2} \mathrm{fm}^{2}$. Also, the large $B(E 1)$ value from the LSSM state at $7.5 \mathrm{MeV}$ is not observed for a specific, experimental state. We note that, in general, there is a large amount of cancellation between the shell-model components of the $E 1$ matrix elements in the PDR region in contrast to states of the GDR (compare the supplement [98] for more details), which introduces a pronounced sensitivity to the Hamiltonian and large uncertainties for the theoretical $B(E 1)$ values. To further highlight this problem, we have excluded the $\left(3 \mathrm{p}_{1 / 2}\right)^{-1}\left(4 \mathrm{~s}_{1 / 2}\right)^{+1}$ and $\left(3 \mathrm{p}_{1 / 2}\right)^{-1}\left(3 \mathrm{~d}_{3 / 2}\right)^{+1}$ contributions to the $B(E 1)$ strengths in Fig.3(i). The strength fragmentation below $7 \mathrm{MeV}$ changes drastically. Missing microscopic configurations or incorrect individual contributions, thus, influence the shape of the $\gamma \mathrm{SF}$.

To study the neutron-skin structure of the $1^{-}$ states with $\left(3 \mathrm{p}_{1 / 2}\right)^{-1}\left(4 \mathrm{~s}_{1 / 2}\right)^{+1},\left(3 \mathrm{p}_{1 / 2}\right)^{-1}\left(3 \mathrm{~d}_{3 / 2}\right)^{+1}$ and other neutron $1 \mathrm{p}-1 \mathrm{~h}$ components, $\mathrm{EDF}+\mathrm{QRPA}$ and EDF + QPM calculations were performed (see 97 for a review). In contrast to [6, 35, 62], single-particle energies were neither determined from nor adjusted to data. Instead, they were directly obtained at the meanfield level from the EDF [97]. Fig.[3(j) presents the QPM+DWBA predictions for $\sigma_{(d, p)}$. Results obtained at the QRPA level and further details are given in the supplement 98]. The QPM also predicts a dominant $\left(3 \mathrm{p}_{1 / 2}\right)^{-1}\left(4 \mathrm{~s}_{1 / 2}\right)^{+1}\left[S_{5.32 \mathrm{MeV}}=0.92\right]$ fragment but ex- 

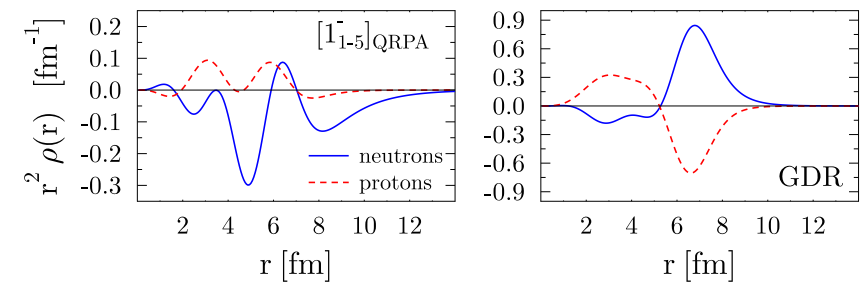

Figure 4. (color online) Summed transition densities for the first five $1_{\mathrm{QRPA}}^{-}$states, which contain the $\left(3 \mathrm{p}_{1 / 2}\right)^{-1}\left(4 \mathrm{~s}_{1 / 2}\right)^{+1}$ and $\left(3 \mathrm{p}_{1 / 2}\right)^{-1}\left(3 \mathrm{~d}_{3 / 2}\right)^{+1}$ components. All five states are dominant neutron $1 \mathrm{p}-1 \mathrm{~h}$ states (compare 98$]$ ). For comparison, the summed transition densities for the GDR are shown.

pects, different from the LSSM and in agreement with experiment, the $\left(3 \mathrm{p}_{1 / 2}\right)^{-1}\left(3 \mathrm{~d}_{3 / 2}\right)^{+1}$ strength to be mainly concentrated in one state $\left[S_{6.12 \mathrm{MeV}}=0.68\right]$. The QPM predicts $\sum \sigma_{(d, p)_{Q P M}}=1676 \mu \mathrm{b}$ below $S_{n}$. However, also the QPM does not fragment the $l=0$ and $l=2$ strength sufficiently to describe the strength above $S_{n}$. While the QPM reproduces the experimental $B(E 1)$ strength distribution around and above $S_{n}$, i.e. where the states' structure becomes more complex [Figs.3(k), (l)], it does not generate sufficiently enhanced strength at lower energies $[$ Fig. $3(\mathbf{m})]$. Due to the doubly magic nature of ${ }^{208} \mathrm{~Pb}$, the $1 \mathrm{p}-1 \mathrm{~h}$ structure of the QRPA phonons dominates the configuration mixing and polarization contributions (compare 98]). In order to improve the comparison with experiment, dynamic effects beyond the static mean field would need to be implemented. As $2 \mathrm{p}-2 \mathrm{~h}$ contributions in the LSSM, multiphonon contributions are small below $8 \mathrm{MeV}$. Interestingly, the $1_{2, Q P M}^{-}$state seems to correspond to the LSSM and experimental $1_{1}^{-}$ state. It has a significant 2-phonon admixture [compare Fig.[3(1)]. Fig. 4 presents the summed transition densities for the first five QRPA $1^{-}$phonons, which contain the $\left(3 \mathrm{p}_{1 / 2}\right)^{-1}\left(4 \mathrm{~s}_{1 / 2}\right)^{+1}$ and $\left(3 \mathrm{p}_{1 / 2}\right)^{-1}\left(3 \mathrm{~d}_{3 / 2}\right)^{+1}$ spectroscopic strengths. The summed transition densities show features which are compatible with the oscillation of the neutron skin [26] and clearly different from the GDR.

In summary, we performed the first extensive study of the single-particle structure of the PDR in ${ }^{208} \mathrm{~Pb}$ based on experimental data. The LSSM and EDF+QPM calculations were able to account for the main features of the $(d, p)$ data. However, both models do not generate enough spectroscopic strengths above $S_{n}$. Such shortcomings could have significant influence on $(n, \gamma)$ rates when determined via surrogate methods using theoretical nuclear-structure input [41, 99, 100]. The extended comparison, including the $\left(p, p^{\prime}\right)_{\text {IAR }}$ data, suggests that the LSSM wave functions might be slightly too complex. At lower energies, the QRPA $1^{-}$phonons might not be sufficiently admixed to several QPM $1^{-}$states. Most $1^{-}$states can, however, not be considered as simple neutron $1 \mathrm{p}-1 \mathrm{~h}$ states as many neutron $1 \mathrm{p}-1 \mathrm{~h}$ excitations contribute to their respective wave function. We pointed out the big cancellation effects between individual $E 1$ matrix elements, which are observed for the PDR in contrast to the GDR. Yet, if the PDR states' structure only contains one or a few $1 \mathrm{p}-1 \mathrm{~h}$ components, individual matrix elements could also not add coherently and generate enhanced $B(E 1)$ strengths. Enhanced strength is observed below $S_{n}$. In contrast to previous claims, the transition densities for the low-lying $1^{-}$states with dominant neutron $1 \mathrm{p}-1 \mathrm{~h}$ character clearly resemble features of a dipole-type neutron-skin oscillation. The present work proves the value of complementary, experimental data on and the theoretical analysis of the PDR's $1 \mathrm{p}-1 \mathrm{~h}$ structure to access the microscopic wave functions. Similar studies will help to further understand the microscopic origin of the low-lying isovector and isoscalar $B(E 1)$ strengths. High-resolution, one-nucleon transfer experiments on stable nuclides in different mass regions, where the change of the underlying single-particle structure can be tracked as both proton and neutron number change, are planned. Further developments at next-generation exotic beam facilities might allow access to the PDR with one-nucleon transfer in inverse kinematics using, e.g., solenoidal spectrometers 101 105.

B.A.B. acknowledges support by the National Science Foundation under Grant No. PHY-1811855. M.Sch. acknowledges financial support by the UK-STFC. N.T. was supported by Extreme Light Infrastructure Nuclear Physics (ELI-NP) Phase II, a project co-financed by the Romanian Government and the European Union through the European Regional Development Fund "the Competitiveness Operational Programme" (1/07.07.2016, COP, ID 1334). A.Z. acknowledges support by the Deutsche Forschungsgemeinschaft under grant ZI 510/9-1. M.Sp. wants to thank K. Kemper and J. Piekarewicz for helpful and inspiring discussions.

* mspieker@fsu.edu

[1] M. Thiel, C. Sfienti, J. Piekarewicz, C. J. Horowitz, and M. Vanderhaeghen, Journal of Physics G: Nuclear and Particle Physics 46, 093003 (201

[2] C. J. Horowitz and J. Piekarewicz, Phys. Rev. Lett. 86, 5647 (2001)

[3] C. J. Horowitz and J. Piekarewicz, Phys. Rev. C 64, 062802 (2001)

[4] F. J. Fattoyev and J. Piekarewicz, Phys. Rev. C 86, 015802 (2012)

[5] F. J. Fattoyev and J. Piekarewicz, Phys. Rev. Lett. 111, 162501 (2013).

[6] A. Tamii, I. Poltoratska, P. von Neumann-Cosel, Y. Fujita, T. Adachi, C. A. Bertulani, J. Carter, M. Dozono, H. Fujita, K. Fujita, K. Hatanaka, D. Ishikawa, M. Itoh, T. Kawabata, Y. Kalmykov, A. M. Krumbholz, E. Litvinova, H. Matsubara, K. Nakanishi, R. Neveling, H. Okamura, H. J. Ong, B. ÖzelTashenov, V. Y. Ponomarev, A. Richter, B. Rubio, 
H. Sakaguchi, Y. Sakemi, Y. Sasamoto, Y. Shimbara, Y. Shimizu, F. D. Smit, T. Suzuki, Y. Tameshige, J. Wambach, R. Yamada, M. Yosoi, and J. Zenihiro, Phys. Rev. Lett. 107, 062502 (2011)

[7] S. Abrahamyan, Z. Ahmed, H. Albataineh, K. Aniol, D. S. Armstrong, W. Armstrong, T. Averett, B. Babineau, A. Barbieri, V. Bellini, R. Beminiwattha, J. Benesch, F. Benmokhtar, T. Bielarski, W. Boeglin, A. Camsonne, M. Canan, P. Carter, G. D. Cates, C. Chen, J.-P. Chen, O. Hen, F. Cusanno, M. M. Dalton, R. De Leo, K. de Jager, W. Deconinck, P. Decowski, X. Deng, A. Deur, D. Dutta, A. Etile, D. Flay, G. B. Franklin, M. Friend, S. Frullani, E. Fuchey, F. Garibaldi, E. Gasser, R. Gilman, A. Giusa, A. Glamazdin, J. Gomez, J. Grames, C. Gu, O. Hansen, J. Hansknecht, D. W. Higinbotham, R. S. Holmes, T. Holmstrom, C. J. Horowitz, J. Hoskins, J. Huang, C. E. Hyde, F. Itard, C.-M. Jen, E. Jensen, G. Jin, S. Johnston, A. Kelleher, K. Kliakhandler, P. M. King, S. Kowalski, K. S. Kumar, J. Leacock, J. Leckey, J. H. Lee, J. J. LeRose, R. Lindgren, N. Liyanage, N. Lubinsky, J. Mammei, F. Mammoliti, D. J. Margaziotis, P. Markowitz, A. McCreary, D. McNulty, L. Mercado, Z.-E. Meziani, R. W. Michaels, M. Mihovilovic, N. Muangma, C. Muñoz Camacho, S. Nanda, V. Nelyubin, N. Nuruzzaman, Y. Oh, A. Palmer, D. Parno, K. D. Paschke, S. K. Phillips, B. Poelker, R. Pomatsalyuk, M. Posik, A. J. R. Puckett, B. Quinn, A. Rakhman, P. E. Reimer, S. Riordan, P. Rogan, G. Ron, G. Russo, K. Saenboonruang, A. Saha, B. Sawatzky, A. Shahinyan, R. Silwal, S. Sirca, K. Slifer, P. Solvignon, P. A. Souder, M. L. Sperduto, R. Subedi, R. Suleiman, V. Sulkosky, C. M. Sutera, W. A. Tobias, W. Troth, G. M. Urciuoli, B. Waidyawansa, D. Wang, J. Wexler, R. Wilson, B. Wojtsekhowski, X. Yan, H. Yao, Y. Ye, Z. Ye, V. Yim, L. Zana, X. Zhan, J. Zhang, Y. Zhang, X. Zheng, and P. Zhu (PREX Collaboration), Phys. Rev. Lett. 108, 112502 (2012).

[8] A. Tamii, P. von Neumann-Cosel, and I. Poltoratska, Eur. Phys. J. A 50, 28 (2013).

[9] C. M. Tarbert, D. P. Watts, D. I. Glazier, P. Aguar, J. Ahrens, J. R. M. Annand, H. J. Arends, R. Beck, V. Bekrenev, B. Boillat, A. Braghieri, D. Branford, W. J. Briscoe, J. Brudvik, S. Cherepnya, R. Codling, E. J. Downie, K. Foehl, P. Grabmayr, R. Gregor, E. Heid, D. Hornidge, O. Jahn, V. L. Kashevarov, A. Knezevic, R. Kondratiev, M. Korolija, M. Kotulla, D. Krambrich, B. Krusche, M. Lang, V. Lisin, K. Livingston, S. Lugert, I. J. D. MacGregor, D. M. Manley, M. Martinez, J. C. McGeorge, D. Mekterovic, V. Metag, B. M. K. Nefkens, A. Nikolaev, R. Novotny, R. O. Owens, P. Pedroni, A. Polonski, S. N. Prakhov, J. W. Price, G. Rosner, M. Rost, T. Rostomyan, S. Schadmand, S. Schumann, D. Sober, A. Starostin, I. Supek, A. Thomas, M. Unverzagt, T. Walcher, L. Zana, and F. Zehr (Crystal Ball at MAMI and A2 Collaboration), Phys. Rev. Lett. 112, 242502 (2014)

[10] B. P. Abbott, R. Abbott, T. D. Abbott, F. Acernese, K. Ackley, et al., The Astrophysical Journal 848, L12 (2017).

[11] B. P. Abbott et al. (LIGO Scientific Collaboration and Virgo Collaboration), Phys. Rev. Lett. 119, 161101 (2017).

[12] F. J. Fattoyev, J. Piekarewicz, and C. J. Horowitz,
Phys. Rev. Lett. 120, 172702 (2018)

[13] D. M. Rossi, P. Adrich, F. Aksouh, H. Alvarez-Pol, T. Aumann, J. Benlliure, M. Böhmer, K. Boretzky, E. Casarejos, M. Chartier, A. Chatillon, D. CortinaGil, U. Datta Pramanik, H. Emling, O. Ershova, B. Fernandez-Dominguez, H. Geissel, M. Gorska, M. Heil, H. T. Johansson, A. Junghans, A. Kelic-Heil, O. Kiselev, A. Klimkiewicz, J. V. Kratz, R. Krücken, N. Kurz, M. Labiche, T. Le Bleis, R. Lemmon, Y. A. Litvinov, K. Mahata, P. Maierbeck, A. Movsesyan, T. Nilsson, C. Nociforo, R. Palit, S. Paschalis, R. Plag, R. Reifarth, D. Savran, H. Scheit, H. Simon, K. Sümmerer, A. Wagner, W. Waluś, H. Weick, and M. Winkler, Phys. Rev. Lett. 111, 242503 (2013).

[14] T. Hashimoto, A. M. Krumbholz, P.-G. Reinhard, A. Tamii, P. von Neumann-Cosel, T. Adachi, N. Aoi, C. A. Bertulani, H. Fujita, Y. Fujita, E. Ganioğlu, K. Hatanaka, E. Ideguchi, C. Iwamoto, T. Kawabata, N. T. Khai, A. Krugmann, D. Martin, H. Matsubara, K. Miki, R. Neveling, H. Okamura, H. J. Ong, I. Poltoratska, V. Y. Ponomarev, A. Richter, H. Sakaguchi, Y. Shimbara, Y. Shimizu, J. Simonis, F. D. Smit, G. Süsoy, T. Suzuki, J. H. Thies, M. Yosoi, and J. Zenihiro, Phys. Rev. C 92, 031305 (2015).

[15] G. Hagen, A. Ekström, C. Forsén, et al., Nature Phys. 12, 186 (2016)

[16] J. Birkhan, M. Miorelli, S. Bacca, S. Bassauer, C. A. Bertulani, G. Hagen, H. Matsubara, P. von NeumannCosel, T. Papenbrock, N. Pietralla, V. Y. Ponomarev, A. Richter, A. Schwenk, and A. Tamii, Phys. Rev. Lett. 118, 252501 (2017).

[17] A. Tonchev, N. Tsoneva, C. Bhatia, C. Arnold, S. Goriely, S. Hammond, J. Kelley, E. Kwan, H. Lenske, J. Piekarewicz, R. Raut, G. Rusev, T. Shizuma, and W. Tornow, Physics Letters B 773, 20 (2017).

[18] X. Roca-Maza and N. Paar, Progress in Particle and Nuclear Physics 101, 96 (2018)

[19] S. Kaufmann, J. Simonis, S. Bacca, J. Billowes, M. L. Bissell, K. Blaum, B. Cheal, R. F. G. Ruiz, W. Gins, C. Gorges, G. Hagen, H. Heylen, A. Kanellakopoulos, S. Malbrunot-Ettenauer, M. Miorelli, R. Neugart, G. Neyens, W. Nörtershäuser, R. Sánchez, S. Sailer, A. Schwenk, T. Ratajczyk, L. V. Rodríguez, L. Wehner, C. Wraith, L. Xie, Z. Y. Xu, X. F. Yang, and D. T. Yordanov, Phys. Rev. Lett. 124, 132502 (2020).

[20] G. Bartholomew, Annu. Rev. Nucl. Sci. 11, 259 (1961).

[21] N. Paar, D. Vretenar, E. Khan, and G. Colò, Reports on Progress in Physics 70, 691 (2007)

[22] D. Savran, T. Aumann, and A. Zilges, Progress in Particle and Nuclear Physics 70, 210 (2013)

[23] Bracco, A., Crespi, F. C. L., and Lanza, E. G., Eur. Phys. J. A 51, 99 (2015)

[24] A. Bracco, E. Lanza, and A. Tamii, Progress in Particle and Nuclear Physics 106, 360 (2019)

[25] J. Piekarewicz, Phys. Rev. C 73, 044325 (2006)

[26] N. Tsoneva and H. Lenske, Phys. Rev. C 77, 024321 (2008)

[27] A. Carbone, G. Colò, A. Bracco, L.-G. Cao, P. F. Bortignon, F. Camera, and O. Wieland, Phys. Rev. C 81, 041301 (2010).

[28] J. Piekarewicz, Phys. Rev. C 83, 034319 (2011).

[29] V. Baran, M. Colonna, M. Di Toro, A. Croitoru, and D. Dumitru, Phys. Rev. C 88, 044610 (2013).

[30] T. Inakura, T. Nakatsukasa, and K. Yabana, 
Phys. Rev. C 84, 021302 (2011)

[31] T. Inakura, T. Nakatsukasa, Phys. Rev. C 88, 051305 (2013)

[32] P.-G. Reinhard and W. Nazarewicz, Phys. Rev. C 87, 014324 (2013)

[33] X. Roca-Maza, X. Viñas, M. Centelles, B. K. Agrawal, G. Colò, N. Paar, J. Piekarewicz, and D. Vretenar, Phys. Rev. C 92, 064304 (2015)

[34] J. Endres, E. Litvinova, D. Savran, P. A. Butler, M. N. Harakeh, S. Harissopulos, R.-D. Herzberg, R. Krücken, A. Lagoyannis, N. Pietralla, V. Y. Ponomarev, L. Popescu, P. Ring, M. Scheck, K. Sonnabend, V. I. Stoica, H. J. Wörtche, and A. Zilges, Phys. Rev. Lett. 105, 212503 (2010)

[35] I. Poltoratska, P. von Neumann-Cosel, A. Tamii, T. Adachi, C. A. Bertulani, J. Carter, M. Dozono, H. Fujita, K. Fujita, Y. Fujita, K. Hatanaka, M. Itoh, T. Kawabata, Y. Kalmykov, A. M. Krumbholz, E. Litvinova, H. Matsubara, K. Nakanishi, R. Neveling, H. Okamura, H. J. Ong, B. Özel-Tashenov, V. Y. Ponomarev, A. Richter, B. Rubio, H. Sakaguchi, Y. Sakemi, Y. Sasamoto, Y. Shimbara, Y. Shimizu, F. D. Smit, T. Suzuki, Y. Tameshige, J. Wambach, M. Yosoi, and J. Zenihiro, Phys. Rev. C 85, 041304 (2012).

[36] D. Savran, V. Derya, S. Bagchi, J. Endres, M. Harakeh, J. Isaak, N. Kalantar-Nayestanaki, E. Lanza, B. Löher, A. Najafi, S. Pascu, S. Pickstone, N. Pietralla, V. Ponomarev, C. Rigollet, C. Romig, M. Spieker, A. Vitturi, and A. Zilges, Physics Letters B 786, 16 (2018).

[37] O. Wieland, A. Bracco, F. Camera, R. Avigo, H. Baba, N. Nakatsuka, T. Aumann, S. R. Banerjee, G. Benzoni, K. Boretzky, C. Caesar, S. Ceruti, S. Chen, F. C. L. Crespi, V. Derya, P. Doornenbal, N. Fukuda, A. Giaz, K. Ieki, N. Kobayashi, Y. Kondo, S. Koyama, T. Kubo, M. Matsushita, B. Million, T. Motobayashi, T. Nakamura, M. Nishimura, H. Otsu, T. Ozaki, A. T. Saito, H. Sakurai, H. Scheit, F. Schindler, P. Schrock, Y. Shiga, M. Shikata, S. Shimoura, D. Steppenbeck, T. Sumikama, S. Takeuchi, R. Taniuchi, Y. Togano, J. Tscheuschner, J. Tsubota, H. Wang, K. Wimmer, and K. Yoneda, Phys. Rev. C 98, 064313 (2018).

[38] S. Goriely, Physics Letters B 436, 10 (1998).

[39] E. Litvinova, H. Loens, K. Langanke, G. MartínezPinedo, T. Rauscher, P. Ring, F.-K. Thielemann, and V. Tselyaev, Nuclear Physics A 823, 26 (2009).

[40] N. Tsoneva, S. Goriely, H. Lenske, and R. Schwengner, Phys. Rev. C 91, 044318 (2015)

[41] A. Larsen, A. Spyrou, S. Liddick, and M. Guttormsen, Progress in Particle and Nuclear Physics 107, 69 (2019)

[42] C. T. Angell, S. L. Hammond, H. J. Karwowski, J. H. Kelley, M. Krtička, E. Kwan, A. Makinaga, and G. Rusev, Phys. Rev. C 86, 051302 (2012)

[43] S. Bassauer, P. von Neumann-Cosel, and A. Tamii, Phys. Rev. C 94, 054313 (2016)

[44] M. Guttormsen, A. C. Larsen, A. Görgen, T. Renstrøm, S. Siem, T. G. Tornyi, and G. M. Tveten, Phys. Rev. Lett. 116, 012502 (2016)

[45] D. Martin, P. von Neumann-Cosel, A. Tamii, N. Aoi, S. Bassauer, C. A. Bertulani, J. Carter, L. Donaldson, H. Fujita, Y. Fujita, T. Hashimoto, K. Hatanaka, T. Ito, A. Krugmann, B. Liu, Y. Maeda, K. Miki, R. Neveling, N. Pietralla, I. Poltoratska, V. Y. Ponomarev, A. Richter, T. Shima, T. Yamamoto, and M. Zweidinger, Phys. Rev. Lett. 119, 182503 (2017).
[46] L. C. Campo, M. Guttormsen, F. L. B. Garrote, T. K. Eriksen, F. Giacoppo, A. Görgen, K. Hadynska-Klek, M. Klintefjord, A. C. Larsen, T. Renstrøm, E. Sahin, S. Siem, A. Springer, T. G. Tornyi, and G. M. Tveten, Phys. Rev. C 98, 054303 (2018)

[47] J. Isaak, D. Savran, B. Löher, T. Beck, M. Bhike, U. Gayer, Krishichayan, N. Pietralla, M. Scheck, W. Tornow, V. Werner, A. Zilges, and M. Zweidinger, Physics Letters B 788, 225 (2019)

[48] N. Simbirtseva, M. Krtička, R. F. Casten, A. Couture, W. I. Furman, I. Knapová, J. M. O'Donnell, G. Rusev, J. L. Ullmann, and S. Valenta, Phys. Rev. C 101, 024302 (2020).

[49] P. Scholz, M. Guttormsen, F. Heim, A. C. Larsen, J. Mayer, D. Savran, M. Spieker, G. M. Tveten, A. V. Voinov, J. Wilhelmy, F. Zeiser, and A. Zilges, Phys. Rev. C 101, 045806 (2020).

[50] D. Savran, M. Babilon, A. M. van den Berg, M. N. Harakeh, J. Hasper, A. Matic, H. J. Wörtche, and A. Zilges, Phys. Rev. Lett. 97, 172502 (2006).

[51] J. Endres, D. Savran, A. M. v. d. Berg, P. Dendooven, M. Fritzsche, M. N. Harakeh, J. Hasper, H. J. Wörtche, and A. Zilges, Phys. Rev. C 80, 034302 (2009)

[52] V. Derya, J. Endres, M. Elvers, M. Harakeh, N. Pietralla, C. Romig, D. Savran, M. Scheck, F. Siebenhühner, V. Stoica, H. Wörtche, and A. Zilges, Nuclear Physics A 906, 94 (2013).

[53] V. Derya, D. Savran, J. Endres, M. Harakeh, H. Hergert, J. Kelley, P. Papakonstantinou, N. Pietralla, V. Ponomarev, R. Roth, G. Rusev, A. Tonchev, W. Tornow, H. Wörtche, and A. Zilges, Physics Letters B 730, 288 (2014)

[54] F. C. L. Crespi, A. Bracco, R. Nicolini, D. Mengoni, L. Pellegri, E. G. Lanza, S. Leoni, A. Maj, M. Kmiecik, R. Avigo, G. Benzoni, N. Blasi, C. Boiano, S. Bottoni, S. Brambilla, F. Camera, S. Ceruti, A. Giaz, B. Million, A. I. Morales, V. Vandone, O. Wieland, P. Bednarczyk, M. Ciemała, J. Grebosz, M. Krzysiek, K. Mazurek, M. Zieblinski, D. Bazzacco, M. Bellato, B. Birkenbach, D. Bortolato, E. Calore, B. Cederwall, L. Charles, G. de Angelis, P. Désesquelles, J. Eberth, E. Farnea, A. Gadea, A. Görgen, A. Gottardo, R. Isocrate, J. Jolie, A. Jungclaus, N. Karkour, W. Korten, R. Menegazzo, C. Michelagnoli, P. Molini, D. R. Napoli, A. Pullia, F. Recchia, P. Reiter, D. Rosso, E. Sahin, M. D. Salsac, B. Siebeck, S. Siem, J. Simpson, P.-A. Söderström, O. Stezowski, C. Theisen, C. Ur, and J. J. ValienteDobón, Phys. Rev. Lett. 113, 012501 (2014).

[55] L. Pellegri, A. Bracco, F. Crespi, S. Leoni, F. Camera, E. Lanza, M. Kmiecik, A. Maj, R. Avigo, G. Benzoni, N. Blasi, C. Boiano, S. Bottoni, S. Brambilla, S. Ceruti, A. Giaz, B. Million, A. Morales, R. Nicolini, V. Vandone, O. Wieland, D. Bazzacco, P. Bednarczyk, M. Bellato, B. Birkenbach, D. Bortolato, B. Cederwall, L. Charles, M. Ciemala, G. D. Angelis], P. Désesquelles, J. Eberth, E. Farnea, A. Gadea, R. Gernhäuser, A. Görgen, A. Gottardo, J. Grebosz, H. Hess, R. Isocrate, J. Jolie, D. Judson, A. Jungclaus, N. Karkour, M. Krzysiek, E. Litvinova, S. Lunardi, K. Mazurek, D. Mengoni, C. Michelagnoli, R. Menegazzo, P. Molini, D. Napoli, A. Pullia, B. Quintana, F. Recchia, P. Reiter, M. Salsac, B. Siebeck, S. Siem, J. Simpson, P.-A. Söderström, O. Stezowski, C. Theisen, C. Ur, J. V. Dobon], and M. Zieblinski, 
Physics Letters B 738, 519 (2014)

[56] E. G. Lanza, A. Vitturi, E. Litvinova, and D. Savran, Phys. Rev. C 89, 041601 (2014)

[57] F. C. L. Crespi, A. Bracco, R. Nicolini, E. G. Lanza, A. Vitturi, D. Mengoni, S. Leoni, G. Benzoni, N. Blasi, C. Boiano, S. Bottoni, S. Brambilla, F. Camera, A. Corsi, A. Giaz, B. Million, L. Pellegri, V. Vandone, O. Wieland, P. Bednarczyk, M. Ciemała, M. Kmiecik, M. Krzysiek, A. Maj, D. Bazzacco, M. Bellato, B. Birkenbach, D. Bortolato, E. Calore, B. Cederwall, G. de Angelis, P. Désesquelles, J. Eberth, E. Farnea, A. Gadea, A. Görgen, A. Gottardo, H. Hess, R. Isocrate, J. Jolie, A. Jungclaus, R. S. Kempley, M. Labiche, R. Menegazzo, C. Michelagnoli, P. Molini, D. R. Napoli, A. Pullia, B. Quintana, F. Recchia, P. Reiter, E. Sahin, S. Siem, P.-A. Söderström, O. Stezowski, C. Theisen, C. Ur, and J. J. Valiente-Dobón, Phys. Rev. C 91, 024323 (2015)

[58] M. Krzysiek, M. Kmiecik, A. Maj, P. Bednarczyk, A. Bracco, F. C. L. Crespi, E. G. Lanza, E. Litvinova, N. Paar, R. Avigo, D. Bazzacco, G. Benzoni, B. Birkenbach, N. Blasi, S. Bottoni, S. Brambilla, F. Camera, S. Ceruti, M. Ciemała, G. de Angelis, P. Désesquelles, J. Eberth, E. Farnea, A. Gadea, A. Giaz, A. Görgen, A. Gottardo, J. Grebosz, H. Hess, R. Isocarte, A. Jungclaus, S. Leoni, J. Ljungvall, S. Lunardi, K. Mazurek, R. Menegazzo, D. Mengoni, C. Michelagnoli, B. Milion, A. I. Morales, D. R. Napoli, R. Nicolini, L. Pellegri, A. Pullia, B. Quintana, F. Recchia, P. Reiter, D. Rosso, M. D. Salsac, B. Siebeck, S. Siem, P.-A. Söderström, C. Ur, J. J. Valiente-Dobon, O. Wieland, and M. Zieblinski, Phys. Rev. C 93, 044330 (2016).

[59] N. Nakatsuka, H. Baba, T. Aumann, R. Avigo, S. Banerjee, A. Bracco, C. Caesar, F. Camera, S. Ceruti, S. Chen, V. Derya, P. Doornenbal, A. Giaz, A. Horvat, K. Ieki, T. Inakura, N. Imai, T. Kawabata, N. Kobayashi, Y. Kondo, S. Koyama, M. KurataNishimura, S. Masuoka, M. Matsushita, S. Michimasa, B. Million, T. Motobayashi, T. Murakami, T. Nakamura, T. Ohnishi, H. Ong, S. Ota, H. Otsu, T. Ozaki, A. Saito, H. Sakurai, H. Scheit, F. Schindler, P. Schrock, Y. Shiga, M. Shikata, S. Shimoura, D. Steppenbeck, T. Sumikama, I. Syndikus, H. Takeda, S. Takeuchi, A. Tamii, R. Taniuchi, Y. Togano, J. Tscheuschner, J. Tsubota, H. Wang, O. Wieland, K. Wimmer, Y. Yamaguchi, K. Yoneda, and J. Zenihiro, Physics Letters B 768, 387 (2017).

[60] F. Crespi, A. Bracco, A. Tamii, N. Blasi, F. Camera, O. Wieland, N. Aoi, D. Balabanski, S. Bassauer, A. S. Brown, M. P. Carpenter, J. J. Carroll, M. Ciemala, A. Czeszumska, P. J. Davies, L. Donaldson, Y. Fang, H. Fujita, G. Gey, T. H. Hoang, N. Ichige, E. Ideguchi, A. Inoue, J. Isaak, C. Iwamoto, D. G. Jenkins, O. H. Jin, T. Klaus, N. Kobayashi, T. Koike, M. Krzysiek, M. K. Raju, M. Liu, A. Maj, D. Montanari, L. Morris, S. Noji, S. G. Pickstone, D. Savran, M. Spieker, G. Steinhilber, C. Sullivan, B. Wasilewska, V. Werner, T. Yamamoto, Y. Yamamoto, X. Zhou, and S. Zhu, Journal of Physics: Conference Series 1014, 012002 (2018)

[61] D. Vretenar, N. Paar, P. Ring, and G. A. Lalazissis, Phys. Rev. C 63, 047301 (2001)

[62] N. Ryezayeva, T. Hartmann, Y. Kalmykov, H. Lenske, P. von Neumann-Cosel, V. Y. Ponomarev, A. Richter, A. Shevchenko, S. Volz, and J. Wambach,
Phys. Rev. Lett. 89, 272502 (2002)

[63] E. Litvinova, P. Ring, V. Tselyaev, and K. Langanke, Phys. Rev. C 79, 054312 (2009).

[64] E. G. Lanza, F. Catara, D. Gambacurta, M. V. Andrés, and P. Chomaz, Phys. Rev. C 79, 054615 (2009).

[65] X. Roca-Maza, G. Pozzi, M. Brenna, K. Mizuyama, and G. Colò, Phys. Rev. C 85, 024601 (2012).

[66] D. Vretenar, Y. F. Niu, N. Paar, and J. Meng, Phys. Rev. C 85, 044317 (2012)

[67] D. Bianco, F. Knapp, N. Lo Iudice, F. Andreozzi, A. Porrino, and P. Vesely, Phys. Rev. C 86, 044327 (2012)

[68] V. Baran, D. I. Palade, M. Colonna, M. Di Toro, A. Croitoru, and A. I. Nicolin, Phys. Rev. C 91, 054303 (2015).

[69] P. Papakonstantinou, H. Hergert, and R. Roth, Phys. Rev. C 92, 034311 (2015)

[70] P. C. Ries, H. Pai, T. Beck, J. Beller, M. Bhike, V. Derya, U. Gayer, J. Isaak, B. Löher, Krishichayan, L. Mertes, N. Pietralla, C. Romig, D. Savran, M. Schilling, W. Tornow, S. Typel, V. Werner, J. Wilhelmy, A. Zilges, and M. Zweidinger, Phys. Rev. C 100, 021301 (2019).

[71] A. Heusler, G. Graw, R. Hertenberger, F. Riess, H.-F. Wirth, R. Krücken, and P. v. Brentano, Phys. Rev. C 75, 024312 (2007).

[72] A. Heusler, T. Faestermann, R. Hertenberger, R. Krücken, H. F. Wirth, and P. von Brentano, Eur. Phys. J. A 46, 17 (2010)

[73] A. Heusler, T. Faestermann, R. Hertenberger, H.-F. Wirth, and P. von Brentano, Phys. Rev. C 89, 024322 (2014).

[74] A. Heusler, H.-J. Glöckner, E. Grosse, C. Moore, J. Solf, and P. von Brentano, Eur. Phys. J. A 50, 92 (2014)

[75] A. Heusler, Eur. Phys. J. A, accepted for publication (2020).

[76] A. Heusler, to be published.

[77] R. Schwengner, R. Massarczyk, B. A. Brown, R. Beyer, F. Dönau, M. Erhard, E. Grosse, A. R. Junghans, K. Kosev, C. Nair, G. Rusev, K. D. Schilling, and A. Wagner, Phys. Rev. C 81, 054315 (2010).

[78] B. A. Brown, Phys. Rev. Lett. 85, 5300 (2000)

[79] A. Heusler, Eur. Phys. J. A 53, 215 (2017).

[80] Maier-Leibnitz-Laboratorium München, https://www.bl.physik. uni-muenchen.de/ (2020).

[81] G. Dollinger and T. Faestermann, Nucl. Phys. News 28, 5 (2018).

[82] H.-F. Wirth et al., Annual Report 2000, Beschleunigerlaboratorium München, 71 (2000), https://www-old.mll-muenchen.de/bl_rep/jb2000/index.html

[83] H.-F. Wirth, Bau eines hochauflösenden Fokalebenendetektors fur den Münchener Q3D-Magnetspektrographen und Untersuchung zur Kernstruktur von ${ }^{129} \mathrm{Te}$, Ph.D. thesis, Technical University Munich (Germany), http://mediatum.ub.tum.de/?id=602907 (2001).

[84] H. A. Enge, Nuclear Instruments and Methods 162, 161 (1979).

[85] M. Martin, Nuclear Data Sheets 108, 1583 (2007)

[86] ENSDF, NNDC Online Data Service, ENSDF database, http://www.nndc.bnl.gov/ensdf/ (2020).

[87] A. Heusler, R. V. Jolos, T. Faestermann, R. Hertenberger, H.-F. Wirth, and P. von Brentano, Phys. Rev. C 93, 054321 (2016).

[88] P. Vold, J. Andreassen, J. Lien, A. Graue, E. Cosman, W. Dünnweber, D. Schmitt, and F. Nüsslin, 
Nuclear Physics A 215, 61 (1973).

[89] M. Schramm, K. H. Maier, M. Rejmund, L. D. Wood, N. Roy, A. Kuhnert, A. Aprahamian, J. Becker, M. Brinkman, D. J. Decman, E. A. Henry, R. Hoff, D. Manatt, L. G. Mann, R. A. Meyer, W. Stoeffl, G. L. Struble, and T.-F. Wang, Phys. Rev. C 56, 1320 (1997)

[90] B. D. Valnion, V. Y. Ponomarev, Y. Eisermann, A. Gollwitzer, R. Hertenberger, A. Metz, P. Schiemenz, and G. Graw, Phys. Rev. C 63, 024318 (2001).

[91] P. D. Kunz and J. R. Comfort, Program CHUCK , unpublished.

[92] F. D. Becchetti and G. W. Greenlees, Phys. Rev. 182, 1190 (1969).

[93] W. W. Daehnick, J. D. Childs, and Z. Vrcelj, Phys. Rev. C 21, 2253 (1980).

[94] A. Debenham, J. Griffith, M. Irshad, and S. Roman, Nuclear Physics A 151, 81 (1970)

[95] B. D. Valnion, Leichtionen-induzierte Anregungen in ${ }^{178} \mathrm{Hf}$ und ${ }^{208} \mathrm{~Pb}$, Ph.D. thesis, Ludwig-MaximiliansUniversität München (Germany) (1998).

[96] D. G. Kovar, N. Stein, and C. K. Bockelman, Nuclear Physics A 231, 266 (1974)

[97] N. Tsoneva and H. Lenske, Phys. Atom. Nuclei 79, 885 (2016)

[98] See Supplemental Material at http://link.aps.org/supplemental/10.1103/yyy.zz.xxxxxx for additional discussion of the theoretical $B(E 1)$ strengths and additional information on the LSSM,
$\mathrm{EDF}+\mathrm{QRPA}$ and $\mathrm{EDF}+\mathrm{QPM}$ wave functions.

[99] A. Ratkiewicz, J. A. Cizewski, J. E. Escher, G. Potel, J. T. Burke, R. J. Casperson, M. McCleskey, R. A. E. Austin, S. Burcher, R. O. Hughes, B. Manning, S. D. Pain, W. A. Peters, S. Rice, T. J. Ross, N. D. Scielzo, C. Shand, and K. Smith, Phys. Rev. Lett. 122, 052502 (2019)

[100] J. E. Escher, J. T. Burke, F. S. Dietrich, N. D. Scielzo, I. J. Thompson, and W. Younes, Rev. Mod. Phys. 84, 353 (2012)

[101] Helical Orbit Spectrometer (HELIOS), https://www.phy .anl.gov/atlas/helios/ (2020).

[102] SOLARIS, https://www.anl.gov/phy/solaris (2020).

[103] SOLARIS White Paper, https://www.anl.gov/sites/www/files/2018-11/solaris_white_ (2018).

[104] ISOLDE Solenoidal Spectrometer (ISS), https://isolde.web.cern.ch/experiments/isolde-solenoidal-s (2020).

[105] T. L. Tang, B. P. Kay, C. R. Hoffman, J. P. Schiffer, D. K. Sharp, L. P. Gaffney, S. J. Freeman, M. R. Mumpower, A. Arokiaraj, E. F. Baader, P. A. Butler, W. N. Catford, G. de Angelis, F. Flavigny, M. D. Gott, E. T. Gregor, J. Konki, M. Labiche, I. H. Lazarus, P. T. MacGregor, I. Martel, R. D. Page, Z. Podolyák, O. Poleshchuk, R. Raabe, F. Recchia, J. F. Smith, S. V. Szwec, and J. Yang, Phys. Rev. Lett. 124, 062502 (2020) 